\title{
Melatonin as a rational alternative in the conservative treatment of resistant hypertension
}

\author{
Fedor Simko ${ }^{1,2,3} \cdot$ Russel J. Reiter ${ }^{4}$ Ludovit Paulis ${ }^{1,5}$
}

Received: 19 July 2019 / Revised: 30 July 2019 / Accepted: 31 July 2019 / Published online: 13 September 2019

(c) The Author(s) 2019. This article is published with open access

Hypertension is the most frequent cardiovascular pathology, afflicting approximately one-third of the population, and its prevalence is rapidly increasing with higher age. The term essential or idiopathic hypertension reflects its unknown etiology. However, decades of research have disclosed the apparent mechanisms leading to essential hypertension and involve endothelial dysfunction, increased oxidative load, neurohumoral imbalance, kidney alterations, or genetic predisposition. What remains truly idiopathic is the level of participation of one or several potential etiologic factors in hypertensive individuals during a particular period of hypertension development. In other words, what renders hypertension as essential is our inability to discern these factors in a particular patient. This is subsequently associated with uncertainty in the choice and dosing of antihypertensive drugs, resulting in poor hypertension control worldwide.

Treatment-resistant hypertension is when blood pressure (BP) remains above the goal despite the use of three antihypertensive drugs of different classes at optimal doses or when target values of BP are achieved only by four or more antihypertensive medications [1]. Resistant hypertension carries greater adverse cardiovascular risk than that of

$\triangle$ Fedor Simko

fedor.simko@fmed.uniba.sk

1 Institute of Pathophysiology, Faculty of Medicine, Comenius University, 81108 Bratislava, Slovakia

2 3rd Department of Internal Medicine, Faculty of Medicine, Comenius University, 83305 Bratislava, Slovakia

3 Institute of Experimental Endocrinology, Biomedical Research Center, Slovak Academy of Sciences, 84505 Bratislava, Slovakia

4 Department of Cellular and Structural Biology, UT Health, San Antonio, TX, USA

5 Institute of Normal and Pathological Physiology, Center of Experimental Medicine, Slovak Academy of Sciences, Bratislava, Slovakia controlled nonresistant hypertension. Based on large clinical trials, the prevalence of resistant hypertension varies from 10 to $30 \%$ and increases with the age of the patients and the duration of hypertension [1]. Although a spectrum of antihypertensive agents is accessible, their inadequate effectiveness, side effects and nonadherence stimulated the introduction of invasive approaches, such as selective renal sympathetic denervation. However, interventional therapy requires a special team and devices, is expensive and results in the loss of counterregulatory adaptive mechanisms against potential hypovolemia; as a result, only a minority of patients with treatment-resistant hypertension are satisfactory candidates for this therapy [1]. Thus, the search after novel conservative treatment options is unremitting.

$\mathrm{N}$-acetyl-5-methoxytryptamine (melatonin) was discovered as the secretory product of the vertebrate pineal gland. It is present in unicellular organisms, fungi, plants, and all animals $[2,3]$. This extensive distribution suggests the hypothetic view that primitive bacteria, which evolved melatonin as an antioxidant defense mechanism, were engulfed by early prokaryotes and developed into mitochondria or chloroplasts. In the phylogenetic development from unicellular to multicellular organisms, melatonin spread to all organisms with subsequent modification of biosynthetic pathways, sites of generation, and functional implications [3]. Melatonin is a pleiotropic molecule that exerts a variety of receptor-dependent and receptorindependent biological effects. The principle receptordependent melatonin action is to coordinate the circadian rhythms of various physiological functions. The regulation and coordination of biological rhythms is based on the mutual interactions between the master clock (the suprachiasmatic nucleus ( $\mathrm{SCN})$ ) in the hypothalamus and several areas in the central nervous system, as well as in peripheral tissues. The information related to light wavelength and intensity is sensed by the retina, transferred to the SCN, and transmitted to the pineal gland, where it controls melatonin production in terms of elevated melatonin secretion during darkness [4]. 
The broad antioxidant effects of melatonin are well recognized. This indoleamine limits oxidative stress both extracellularly and intracellularly by a variety of mechanisms, such as direct radical scavenging, stimulating the activity and expression of antioxidative enzymes, supporting glutathione synthesis and recycling, protecting other antioxidants and downregulating pro-oxidant enzymes. Melatonin also improves mitochondrial electron transport and energetic gain and modifies inflammation and apoptosis. These subcellular protective actions of melatonin result in a number of potentially beneficial actions in various systems, including a BP-reducing effect [4]. Whether melatonin can exert protection in the treatment of resistant hypertension has not been investigated.

The two-kidney one-clip model of hypertension, introduced by Goldblatt almost a century ago, is induced by the narrowing of one renal artery inducing unilateral renal ischemia with the activation of the renin-angiotensin system (RAS). Recent experiments disclosed the complex nature of this model, represented by renal hypoperfusion, oxidative stress stimulation, and sympathetic nervous system (SNS) and RAS activation. The crosstalk between the brain and kidney is impressive [5]. Kidney efferent sympathetic fibers stimulate renal artery vasoconstriction, renin release from juxtaglomerular cells and sodium and water reabsorption by the tubular system. Ischemic kidney (IK) afferent nerves modulate neurotransmission in brain areas involved in cardiovascular control. The hypothalamic paraventricular nucleus (PVN) and brainstem rostral ventrolateral medulla (RVLM) are supposedly influenced by tissue and/or circulating angiotensin II (Ang II), resulting in a sequence of events in transcriptional factor synthesis that alter the passage of impulses in baroreflex sympathetic neurons [6]. Indeed, angiotensin type 1 receptors (AT1R) and oxidative stress were shown to be increased in RVLM along with baroreflex disturbance and BP enhancement [5]. Moreover, the renal density of AT1R and urinary angiotensinogen was increased in the $2 \mathrm{~K} 1 \mathrm{C}$ model [5]. Although $2 \mathrm{~K} 1 \mathrm{C}$ is a model of secondary hypertension, due to its complex pathophysiology, this model bears considerable resemblance to resistant hypertension in clinical conditions.

The attractive and stimulating recent works of Nishi et al. $[5,7]$. showed that alterations in the $2 \mathrm{~K} 1 \mathrm{C}$ model were partly reversed either by unilateral sympathetic denervation or by melatonin treatment. These authors demonstrated that the unilateral renal denervation of IK reduced the mean arterial BP and renal and splanchnic activity of SNS (rSNS, sSNS) in the contralateral kidney, with a reduction in AT1R and oxidative stress in IK and kidney protection in terms of preserving structure and reducing proteinuria. Moreover, oxidative stress and the AT1 and AT2 receptor numbers were reduced in the PVN and RVLM in the 2K1C model [5]. As such, these results support the idea that $2 \mathrm{~K} 1 \mathrm{C}$ hypertension is renovascular in its etiology but neurogenic in its mechanisms. Subsequently, the hypothesis of central modulation/treatment of this type of hypertension emerges. Indeed, gavage treatment by melatonin ( $30 \mathrm{mg} / \mathrm{kg} /$ day) for 15 days after the 5 -week clipping of the left renal artery, prominently reduced mean arterial pressure (by $45 \mathrm{mmHg}$ ), attenuated sympathoexcitation to the IK, and normalized cardiac baroreflex gain and rSNS answer. Renal protection was reflected by the reduction in oxidative stress in IK and the lowering of proteinuria [7]. Yet, the study still leaves a gap in the knowledge. The principal unanswered question is where the primary action of melatonin took place. The central modulation of sympathetic outflow remains the most plausible option. However, a direct renoprotective effect of melatonin with subsequent reduced afferent stimulation from the kidney could also be postulated. Moreover, to document the potential interference of melatonin with the RAS, the Ang II levels and AT1R number in the brain and kidney should be investigated. It might also be valuable to determine the serum aldosterone level or AT1R number in the renal cortex, since aldosterone seems to play an important role in resistant hypertension. Furthermore, serum or tissue melatonin concentrations would indicate whether exogenous melatonin compensated for the potential melatonin deficit. These areas remain a challenge for future experiments.

In terms of the recent experimental findings and depending on the answers to the open questions, the following mechanisms could emerge (Fig. 1):

First, endogenous melatonin production in the pineal gland is bound with the SNS. The axons of neurons in the PVN project to the preganglionic sympathetic neurons of the cervical intermediolateral cell column controlling vascular tone and arterial BP. The sympathetic impulses from intermediolateral column cells simultaneously project to the superior cervical ganglia to eventually stimulate melatonin production by the pineal gland via beta and alpha-1 adrenoceptor activation. Melatonin may have a negative feedback effect on the sympathetic system by GABA-ergic inhibitory signaling on PVN via $\mathrm{SCN}$, the action of which may be potentiated by nitric oxide. Thus, endogenous melatonin may represent a counterregulatory mechanism against excessive sympathetic stimulation [8, 9]. Moreover, Melo et al. [10]. revealed in $2 \mathrm{~K} 1 \mathrm{C}$ rats that the AT1R inhibitor losartan or the activation of GABAergic receptor in the commissural nucleus (cNTS), a part of the NTS, reduced ROS, inflammation and microglia in the cNTS along with BP reduction. Since exogenous melatonin readily crosses the brain barrier [11], it may inhibit the NTS by augmenting GABAergic neurotransmission and by its extraordinary antioxidative actions to attenuate also other sympathetic loci regulating BP. Given that alpha- and beta- blockers are seldomly used because of side effects or metabolism 


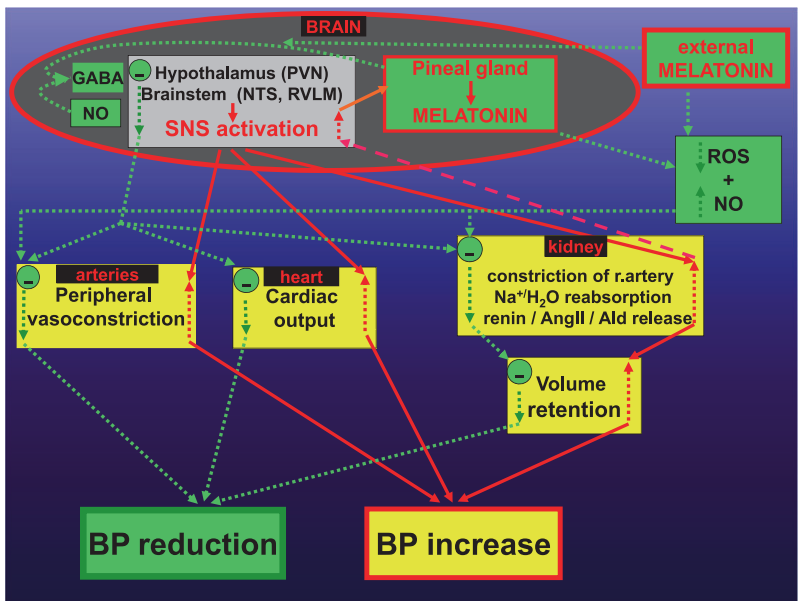

Fig. 1 The paraventricular nucleus (PVN) of the hypothalamus and rostral ventrolateral medulla (RVLM) and nucleus of the solitary tract (NTS) of the brainstem participate in the activity of the sympathetic nervous system (SNS). The sympathetic impulses from the intermediolateral cell column increase the peripheral vascular tone and cardiac output and modify kidney function in terms of volume retention, all of which result in an enhancement of blood pressure (BP). Kidney alterations can stimulate SNS. Endogenous or exogenous melatonin may attack two principal factors of resistant hypertension: SNS and circulating volume overload. Endogenous melatonin, which is stimulated by sympathetic flow via superior cervical ganglia, exerts a negative feedback effect on SNS via $\gamma$-aminobutyric acid (GABA) inhibitory signaling on PVN, reducing the sympathetic outflow to peripheral arteries, heart and kidneys. Exogenous melatonin may carry analogical action in the SNS and can also increase the bioavailability of nitric oxide (NO) with peripheral and renal artery vasodilatation, resulting in $\mathrm{BP}$ reduction

disruptions, melatonin's sympatholytic action could be of principal value.

Second, melatonin exerts protection in various models of kidney damage, including hypertensive and diabetic nephropathy, renal damage induced by toxic substances or invasive interventions [2]. RAS seems to play an important role in chronic kidney disturbances. The impaired melatonin secretion is associated with increased nighttime activation of local-renal RAS and renal damage in patients with chronic kidney disease. Moreover, the administration of melatonin improved structure and function in animal models of renal damage, which was associated with attenuation of intrarenal RAS activation and oxidative stress [12]. Preserving kidney function could attenuate the volume overload and hyperdynamic mechanisms of resistant hypertension development.

Third, melatonin, via its antioxidant and antiproliferative effects [13], may improve the endothelium-dependent and smooth musculature-dependent dilation of arterioles, resulting in a reduction of peripheral vascular resistance.

Since the BP threshold for starting antihypertensive therapy was reduced to $\geq 130 / 80 \mathrm{mmHg}$ in the AHA guidelines, the prevalence of patients with treatmentresistant hypertension is likely to increase. Thus, finding a novel therapeutic option is a challenging issue. Commonly used therapeutic regimes involve volume reducing agents, inhibitors of the formation or effects of Ang II, and calcium channel blockers [1]. While the treatment-resistant hypertension often contains a central neurogenic pathomechanical component, the above postulated mechanisms of action of melatonin could find their application in its treatment. Based on the 2K1C model and other models of hypertension, melatonin triggers sympatholytic and bradycardic effects [9] and, due to its apparent antiproliferative action [13], this hormone even offers nephro- and cardioprotection beyond BP reduction. Therefore, melatonin becomes an essential candidate to be investigated for the treatment of resistant hypertension.

Acknowledgements This work was supported by following grants for scientific research: VEGA 1/0035/19, VEGA 2/0112/19, VEGA 1/ 0127/17.

\section{Compliance with ethical standards}

Conflict of interest The authors declare that they have no conflict of interest.

Publisher's note: Springer Nature remains neutral with regard to jurisdictional claims in published maps and institutional affiliations.

Open Access This article is licensed under a Creative Commons Attribution 4.0 International License, which permits use, sharing, adaptation, distribution and reproduction in any medium or format, as long as you give appropriate credit to the original author(s) and the source, provide a link to the Creative Commons license, and indicate if changes were made. The images or other third party material in this article are included in the article's Creative Commons license, unless indicated otherwise in a credit line to the material. If material is not included in the article's Creative Commons license and your intended use is not permitted by statutory regulation or exceeds the permitted use, you will need to obtain permission directly from the copyright holder. To view a copy of this license, visit http://creativecommons. org/licenses/by/4.0/.

\section{References}

1. Carey RM, Calhoun DA, Bakris GL, Brook RD, Daugherty SL, Dennison-Himmelfarb CR, et al. Resistant hypertension: detection, evaluation, and management: a scientific statement from the American Heart Association. Hypertension. 2018;72:e53-90.

2. Hrenak J, Paulis L, Repova K, Aziriova S, Nagtegaal EJ, Reiter $\mathrm{RJ}$, et al. Melatonin and renal protection: novel perspectives from animal experiments and human studies (review). Curr Pharm Des. 2015;21:936-49.

3. Zhao D, Yu Y, Shen Y, Liu Q, Zhao Z, Sharma R, et al. Melatonin synthesis and function: evolutionary history in animals and plants. Front Endocrinol 2019;10:249.

4. Reiter RJ, Tan DX, Rosales-Corral S, Galano A, Zhou XJ, Xu B. Mitochondria: central organelles for melatonin's antioxidant and 
anti-aging actions. Molecules. 2018;23:E509. https://doi.org/10. 3390/molecules23020509

5. Nishi EE, Lopes NR, Gomes GN, Perry JC, Sato AYS, NaffahMazzacoratti MG, et al. Renal denervation reduces sympathetic overactivation, brain oxidative stress, and renal injury in rats with renovascular hypertension independent of its effects on reducing blood pressure. Hypertens Res. 2019;42:628-40.

6. Braga VA. Is the commissural nucleus of the solitary tract essential for the aintenance of renovascular hypertension? A putative role for the carotid bodies. Hypertens Res. 2019;42:749-51.

7. Nishi EE, Almeida VR, Amaral FG, Simon KA, Futuro-Neto HA, Pontes RB, et al. Melatonin attenuates renal sympathetic overactivity and reactive oxygen species in the brain in neurogenic hypertension. Hypertens Res. 2019. https://doi.org/10.1038/ s41440-019-0301-z (Epub ahead of print).

8. Paulis L, Simko F. Blood pressure modulation and cardiovascular protection by melatonin: potential mechanisms behind. Physiol Res. 2007;56:671-84.
9. Simko F, Baka T, Paulis L, Reiter RJ. Elevated heart rate and nondipping heart rate as potential targets for melatonin: a review. $\mathrm{J}$ Pineal Res. 2016;61:127-37.

10. Melo MR, Gasparini S, Speretta GF, Silva EF, Rodrigues Pedrino G, Menani JV, et al. Importance of the commissural nucleus of the solitary tract in renovascular hypertension. Hypertens Res. 2019;42:587-97.

11. Reiter RJ, Tan DX, Kim SJ, Cruz MH. Delivery of pineal melatonin to the brain and SCN: role of canaliculi, cerebrospinal fluid, tanycytes and Virchow-Robin perivascular spaces. Brain Struct Funct. 2014;219:1873-87.

12. Ohashi $\mathrm{N}$, Ishigaki $\mathrm{S}$, Isobe $\mathrm{S}$. The pivotal role of melatonin in ameliorating chronic kidney disease by suppression of the renin-angiotensin system in the kidney. Hypertens Res. 2019; 42:761-8.

13. Simko F, Pechanova O. Remodelling of the heart and vessels in experimental hypertension: advances in protection. J Hypertens. 2010;28(Suppl 1):S1-6. 